\title{
La teleriabilitazione nel bambino con patologia neurologica cronica: prendersi cura a distanza è possibile
}

\author{
Camilla Garzaniti* , Martina Fornaro**, Martina Casprini*, Elena Guidi*, \\ Monica Giorgioni* , Giustino Melideo*, Enrico Valletta** \\ * UO Neuropsichiatria dell'infanzia e dell'adolescenza; \\ ** UO di Pediatria, Ospedale G.B. Morgagni - L. Pierantoni, AUSL della Romagna, Forlì
}

La pandemia di SARS-Cov-2 ha avuto un profondo impatto sul servizio sanitario nazionale impegnando anche i servizi territoriali nella sorveglianza domiciliare e nel mantenimento della presa incarico dei pazienti con patologie croniche.

Le norme restrittive introdotte dal mese di marzo 2020 hanno determinato una situazione assai sfavorevole per il mondo dell'infanzia e, in particolare, per i bambini e gli adolescenti con patologie neuropsicologiche croniche a media-alta complessità. Il progressivo inasprirsi dei provvedimenti ha presto portato al cosiddetto "lockdown", con la chiusura delle scuole e l'obbligo di spostamento esclusivamente per motivi urgenti. I nostri servizi hanno dovuto sospendere le consuete attività ambulatoriali programmate, ferma restando, comunque, la disponibilità ad accogliere le situazioni che richiedessero un intervento tempestivo. La possibilità che questa contingenza potesse trasformarsi in un ulteriore fattore di rischio per la salute di bambini e adolescenti già portatori di importanti fragilità è stata la nostra prima preoccupazione [1-3]. L'obiettivo su cui ci siamo concentrati è stato quello di individuare soluzioni coerenti con la normativa, ma in grado di assicurare prossimità alle famiglie e continuità ai percorsi di cura già avviati. L'elaborazione di nuove strategie di assistenza e riabilitazione "da remoto" ha dovuto fare i conti con le consuetudini operative dei professionisti, con la scarsità delle esperienze disponibili in letteratura e, d'altra parte, con la necessità di offrire in tempi brevi la prosecuzione dell'assistenza, se pure a distanza [4-8]. Gli strumenti scelti sono stati diversi, si sono affinati nel tempo e sono stati volti a offrire le risorse tecniche e metodologiche per proseguire i trattamenti indispensabili. Si è cercato, infine, di mantenere saldo il principio della multidisciplinarietà in tutti i casi nei quali la richiesta fosse ineludibile.

L'esperienza descritta ripercorre quanto fatto, in termini di teleriabilitazione, nel corso della recente emergenza sanitaria, prefigurando, altresì, l'impiego delle nuove modalità di approccio "da remoto" anche in condizioni di normalità operativa post post emergenziale.

\section{Come l'emergenza ha modificato il nostro lavoro}

All'interno dell'UO di Neuropsichiatria dell'infanzia e dell'adolescenza (UONPIA) operano, con attività prevalentemente ambulatoriale e in maniera coordinata, neuropsichiatri infantili, psicologi, fisioterapisti, logopedisti, educatori e infermieri. La presa in carico del bambino-adolescente può avvenire da parte del singolo professionista o come équipe multidisciplinare. Nell'ambito del progetto riabilitativo, le attività individuali o di gruppo si svolgono con frequenza settimanale o bisettimanale, in accordo con le linee guida più aggiornate. I setting possono anche essere diversi da quello classico ambulatoriale: in ospedale, a domicilio, a scuola o presso centri educativi.

Transitare da un'attività riabilitativa "in presenza" a una "da remoto" ha richiesto di assumere come presupposti indispensabili la disponibilità dei necessari strumenti tecnico-informatici, l'autonomia informatica dei professionisti, la definizione degli obiettivi e una modalità condivisa di monitoraggio del lavoro svolto. Anche il coordinamento dell'attività tra gli operatori è avvenuto prevalentemente con mezzi informatici.

Gli strumenti scelti per la teleriabilitazione rispondevano ai requisiti della evidence based practice $[9,10]$. Sono stati utilizzati software gratuiti per videoconferenze, applicazioni di messaggistica ed email per la condivisione dei programmi di trattamento, piattaforme riabilitative (preesistenti o create ex novo) per il trattamento dei disturbi del linguaggio e dell'apprendimento. I principi ispiratori sono stati quelli della family centered care, ancor più fondanti in questo periodo nel quale la famiglia è stata protagonista non solo del progetto e della sua realizzazione, ma anche della messa in pratica di atti terapeutici normalmente gestiti dai riabilitatori [11].

È stata prevista una duplice modalità di interazione: sincrona o asincrona [12]. Nella modalità sincrona, l'operatore comunica direttamente con il bambino/ragazzo, attraverso lo strumento tecnologico, proponendo l'attività riabilitativa. L'intervento asincrono si adotta quando non è possibile avere il contatto diretto con il paziente e, con l'intermediazione della famiglia, si propongono le indicazioni personalizzate per il trattamento, si guidano le azioni, vengono monitorate e condivise le difficoltà, modificando in itinere le proposte. $\mathrm{Al}$ termine della videochiamata il riabilitatore rivede il programma, effettua gli aggiustamenti necessari, li condivide con la famiglia tramite email e attende un feedback attraverso filmati o fotografie. Sono stati progettati e realizzati videotutorial personalizzati per illustrare le attività riabilitative e, in previsione di una ripresa dei trattamenti in presenza, per abituare i bambini più piccoli o con difficoltà relazionali ai presidi individuali di protezione che i riabilitatori avrebbero dovuto indossare.

Per le situazioni di maggiore disagio o nelle quali non vi era disponibilità di strumenti informatici, è stato previsto un periodico contatto telefonico e la possibilità di un intervento ambulatoriale nei casi di urgente criticità.

\section{Il lavoro negli specifici campi di applicazione}

Ogni professionista ha riadattato le proprie prestazioni alle nuove modalità, tenendo conto sia delle differenze di età dei pazienti sia delle diverse aree di intervento e, spesso, della necessità di un trattamento multidisciplinare.

La logopedista (Box 1) ha utilizzato diversi materiali (informatici e non) a seconda della fascia di età (3-5 anni e età scolare) e delle difficoltà del singolo bambino (disturbi di linguaggio espressivo e recettivo, 
disturbi dell'apprendimento, sordità). Generalmente, sono state effettuate sedute di riabilitazione settimanali o quindicinali mediante videochiamata con computer o smartphone. Sia per i disturbi di linguaggio che per i disturbi di apprendimento è stato possibile condividere lo schermo e il materiale scelto con il paziente: sono state utilizzate piattaforme (DIDALabs, RIDInet) (Tabella 1), con possibilità di accesso immediato o in differita, e preparati file animati con Powerpoint. Nei bambini più piccoli e nei casi di sordità, con difficoltà specifiche di linguaggio e dell'ambito fonetico-fonologico, si è lavorato sui processi $\mathrm{da}$ correggere in un contesto ludico strutturato, ampliando parallelamente il lessico e potenziando le competenze morfosintattiche, metafonologiche e le funzioni esecutive. Con i bambini in età scolare si è mirato a potenziare gli aspetti della letto-scrittura con attività specifiche di lettura tachistoscopica, dettato, riconoscimento globale e altre attività applicabili in remoto.

Nella modalità asincrona, utilizzata per i bambini con particolari difficoltà, la logopedista ha effettuato colloqui telefonici e/o videochiamate con le famiglie per confrontarsi sugli obiettivi e sulle modalità proposti e svolgere attività di counselling. Periodicamente è stato inviato materiale dedicato via email, richiedendo alla famiglia un riscontro settimanale tramite foto o video. Si è lavorato, quindi, sugli aspetti relativi allo sviluppo della comunicazione in contesto domiciliare mediante specifici approcci (PECS, agenda visiva, comunicatori) e monitorando gli aspetti fondamentali della deglutizione (training masticatorio, svezzamento, manipolazione del cibo).

Gli educatori professionali (Box 2), inseriti nella UONPIA, sono esclusivamente dedicati al trattamento dei bambini con disturbo dello spettro autistico. Ogni intervento educativo è stato pensato e progettato partendo da un'analisi dei bisogni già noti e su quelli emergenti, espressi dalla famiglia, riguardo alla gestione del paziente nel contesto domiciliare.

Nella modalità sincrona si è interagito direttamente davanti allo schermo del PC con il bambino o ragazzo che, assieme al genitore, ha seguito le indicazioni fornite dall'educatore. Questa modalità è stata utilizzata con i pazienti che potevano garantire la condivisione dello sguardo oculare (capacità di guardare e riconoscere l'altro allo schermo), la capacità di imitazione e tempi di attenzione prolungati (20-30 minuti). Nella modalità asincrona sono stati inviati via email alle famiglie dei file personalizzati con la descrizione delle attività da svolgere e il materiale da utilizzare. Considerando le limitazioni

BOX 1

Simone ha 5 anni ed è affetto da sindrome di Pallister-Killian. L'équipe curante è composta da fisioterapista (FT), logopedista (LP) e neuropsichiatra infantile (NPI). Simone è seguito dall'Ambulatorio del bambino complesso della Pediatria dell'ospedale di Forlì e dal Centro regionale ausili di Bologna. II progetto riabilitativo prevede due sedute settimanali di fisioterapia e una di logopedia che affrontano le diverse aree di intervento: cognitiva, relazionale e linguistico-comunicativa, senso-percettiva, motoria grossolana e fine e della funzionalità oro-buccale. I trattamenti di Simone in presenza sono stati sospesi già nel febbraio 2020 e sono stati sostituiti da supervisioni settimanali in remoto, svolte in équipe (LP e FT) con i genitori con videochiamate in modalità asincrona e colloqui telefonici. È stato così possibile mostrare modalità e approcci relativi al controllo e all'adattamento degli ausili comunicativi mediante l'utilizzo di attività molto gradite a Simone (strumenti musicali, scatole rumorose, video musicali, ecc.) grazie alle quali si è potuto allenarlo alla richiesta e al condizionamento causa-effetto sollecitando la sua attivazione motoria (premere il tasto "ancora"). Sempre in remoto, è stata gestita la fornitura di due ausili comunicativi (tasto BigBeamer Slat Wireless e comunicatore simbolico mono-messaggio) consegnati in pieno lockdown. II buon esito di questi passaggi ha richiesto un continuo confronto telefonico e in videochiamata all'interno dell'équipe. È proseguita regolarmente l'attività di sostegno alla famiglia per monitorare gli adattamenti posturali (statica, swash, costruzione di un'altalena adattata) e la risoluzione di specifiche difficoltà di alimentazione e manipolazione del cibo. Sono state suggerite e realizzate dai genitori attività ludiche per favorire la manipolazione del cibo, la stimolazione percettiva e l'esplorazione tattile e orale mediante scatole rumorose piene di cereali e legumi, collane e bastoncini costruiti col cibo e frullati di frutta.

In modalità asincrona, sono state illustrate strategie di svezzamento dal biberon, utilizzando utensili scelti e condivisi mediante file esplicativi e foto via mail. I genitori hanno collaborato attivamente e con entusiasmo e hanno inviato feedback (foto, video, note audio) delle attività realizzate, mostrando le modalità di esecuzione e il loro esito.

\section{TABELLA 1. Glossario dei termini}

Agenda visiva: strumento visivo personalizzato che favorisce la comunicazione e l'apprendimento visivo utilizzato comunemente nei disturbi dello spettro autistico.

Competenze metafonologiche: capacità di percepire, analizzare e manipolare i suoni del linguaggio parlato. Competenze morfosintattiche: capacità di comprendere e costruire le strutture frasali della lingua italiana. Comunicatori: ausili/dispositivi elettronici di comunicazione aumentativa alternativa per supportare la comunicazione non verbale.

DIDALabs: piattaforma online che propone attività didattiche (letto-scrittura e matematica) multimediali, interattive e personalizzate da poter svolgere a distanza.

Early Start Denver Model: strumento di monitoraggio per definire gli obiettivi di trattamento in età precoce. PECS: Picture Exchange Communication System (sistema di comunicazione per scambio di immagini), è una forma di comunicazione aumentativa alternativa prodotta dalla Pyramid Educational Consultants, utilizzata comunemente come aiuto per la comunicazione per i bambini con disturbo dello spettro autistico o ritardo mentale.

RIDInet: piattaforma di teleriabilitazione ideata per la riabilitazione online dei disturbi specifici di apprendimento e dei bisogni educativi speciali.

Swash: ortesi per bacino e anche che favorisce il corretto allineamento dei capi articolari con l'obiettivo di evitare, o quantomeno posticipare, la lussazione d'anca.

Tachistoscopia: tecnica di riabilitazione e potenziamento delle difficoltà di lettura, consistente nella lettura rapida e temporizzata di parole.

Vineland Adaptive Behaviour Scales: strumento di monitoraggio per la valutazione funzionale che considera il contesto di vita della persona (famiglia, scuola, tempo libero). Vedi Programma regionale integrato per l'assistenza territoriale alle persone con disturbo dello spettro autistico (PRIA): obiettivi 2016-2018.

degli spostamenti imposte alle famiglie, si è pensato di proporre giochi già presenti nelle case o che fosse possibile costruire con materiali di recupero. Una volta alla settimana i genitori inviavano all'educatore un feedback (foto/video) sulle attività effettuate per verificare il raggiungimento degli obiettivi. Ogni intervento educativo è stato progettato utilizzando materiali adeguati all'età e al funzionamento cognitivo del paziente. Per i bambini di 12-36 mesi si è utilizzato l'Early Start Denver Model, preparando il materiale per specifiche aree: attenzione condi- visa, abilità sociali, imitazione, cognizione, gioco autonomo e rappresentazionale, motricità fine, motricità grossolana, autonomie (mangiare, vestirsi, lavarsi, lavori di casa). Per l'intervento rivolto ai ragazzi (8-14 anni) si è utilizzato le Vineland Adaptive Behaviour Scales (Tabella 1).

L'attività della fisioterapista (Box 3$)$ in modalità sincrona (videochiamata) ha richiesto, come presupposti, un'età e un grado di compliance sufficienti a consentirle di interagire direttamente con il ragazzo o la collaborazione dei genitori. Il fisioterapista ha dapprima impostato il setting scegliendo il 
luogo e gli attrezzi più adeguati per la seduta. Quindi, ha proposto:

- esercizi nelle aree compatibili con la nuova modalità di trattamento (rinforzo e allungamento muscolare, stretching, balance, coordinazione, equilibrio, stabilità del core), controllando e correggendo l'attività durante l'esecuzione;

- attività per la facilitazione dell'autonomia quotidiana (vestirsi-svestirsi, passaggi posturali, gestione degli ausili per la mobilità), consigliando su come adattarsi alla prolungata permanenza negli spazi domestici;

- esercizi di fisioterapia respiratoria;

- attività di manipolazione e manualità fine utilizzando giochi e convertendo oggetti di uso quotidiano;

- attività volte al raggiungimento o alla stabilizzazione di una funzione in emergenza (posizione seduta, quadrupedica, stazione eretta, deambulazione).

La modalità asincrona è stata utilizzata quando non fosse possibile ottenere una compliance soddisfacente o come integrazione alla modalità sincrona. Il terapista, in accordo con il genitore, ha stilato un programma di attività prendendo in considerazione il setting domestico, l'esercizio terapeutico, l'attività di gioco condiviso, il riadattamento di oggetti presenti a domicilio e la costruzione di materiali ad hoc. I programmi sono stati inviati tramite email e, a cadenza settimanale, il genitore ha inviato feedback tramite foto o video; i colloqui telefonici regolari hanno permesso il confronto sulle attività e il monitoraggio dei programmi concordati.

\section{Qualche valutazione sull'attività} di teleriabilitazione svolta

Da gennaio 2020 all'inizio del lockdown i pazienti, seguiti con trattamento riabilitativo in presenza, erano 423; nel periodo successivo è stato possibile proseguire il trattamento nell'88\% (374) dei casi. Con la progressiva riapertura degli ambulatori in presenza, il numero dei bambini seguiti attivamente è risalito a 408 (Tabella 2). Particolare attenzione è stata rivolta ai bambini con disturbo del linguaggio e autismo per i quali si è riusciti a mantenere la continuità terapeutica da remoto nel $93 \%$ (228 su 245) dei casi. Il complesso delle attività di telemedicina $(3279$ prestazioni) effettuate nel periodo del lockdown è sintetizzato nella Tabella 3.

I dati relativi alle prestazioni fanno emergere alcune peculiarità operative proprie delle singole specialità. La logopedista ha largamente impiegato sia le videochiamate, sia i colloqui telefonici di confronto con la famiglia sulle attività consigliate. Le caratteristiche di questi pazienti hanno permes-

BOX 2

Roberto è un bambino di 9 anni con autismo. La sua équipe terapeutica è composta da FT, LP, educatore (ED), psicologo (PSI) e NPI. Esegue con cadenza settimanale trattamenti logopedici e fisioterapici. L'intervento educativo ha previsto trattamenti al domicilio del paziente e un trattamento di gruppo per lo sviluppo delle abilità sociali iniziato a gennaio 2020. Sospeso il gruppo, si è proseguito il sostegno alla famiglia proponendo la teleriabilitazione in modalità sincrona e asincrona. II computer è stato uno strumento di mediazione efficace e di alta motivazione per Roberto. C'è stata una buona aderenza alla proposta, un aumento del tempo di concentrazione rispetto al trattamento in presenza, una maggiore collaborazione e puntualità agli incontri programmati.

La modalità sincrona è stata utilizzata da LP, ED e PSI. L'intervento logopedico è stato mirato all'acquisizione del meccanismo di lettura (corrispondenza grafema e fonema, riconoscimento di sillabe e decodifica bisillabica) e dei digrammi, trigrammi e gruppi di consonanti. L'intervento educativo è stato organizzato mantenendo l'aggancio relazionale, facendosi raccontare cosa avesse fatto durante la settimana e mostrare i giochi per lui più importanti. Si è lavorato sul mantenimento degli obiettivi programmati utilizzando le Vineland Adaptive Behaviour Scales (scala della comunicazione - subscala espressione). Si è proposto di utilizzare Google Maps con l'impostazione satellitare per aiutarlo nell'orientamento; Roberto ha così potuto guardare in autonomia, nella modalità "street view", la Torre Eiffel e Parigi esprimendo il desiderio di viaggiare insieme alla famiglia. Nei colloqui telefonici è stato proposto di dare al ragazzo un ruolo di responsabilità all'interno del nucleo familiare, per gratificarlo e motivarlo nelle autonomie e nella gestione delle attività domestiche (lavarsi i denti la mattina, vestirsi da solo, lavare la tazza dopo la colazione, stendere i panni, apparecchiare la tavola, lavare i piatti). Per ogni attività svolta, i genitori hanno inviato materiale foto e video utile per il monitoraggio di quanto realizzato.

In modalità asincrona, si è preparato e concordato il materiale con l'équipe e con la famiglia. La FT ha proposto attività relative alle macroaree individuate (gioco condiviso, prassia, grosso-motorio) integrando con videotutorial degli esercizi da eseguire (esercizi aerobici, per saltare, lanciare la palla correttamente, salto monopodalico, coordinazione dinamica). La famiglia è stata coinvolta in attività di gioco condiviso (gioco con la palla, alternanza del turno, gioco motorio con il fratello minore). I genitori hanno confermato l'importanza che ha avuto per loro il contatto costante con l'équipe curante e che, per Roberto, era diventato fondamentale sapere che c'era chi lo pensava proponendogli strategie su come vivere la quotidianità insieme. Roberto ha oggi sviluppato nuove abilità e competenze: apparecchia la tavola, va in bicicletta in autonomia senza sostegno e aiuta a cucinare.

\section{BOX 3}

Antonio è un bambino di 3 anni con tetraplegia spastica e la sua équipe terapeutica include NPI, fisiatra e FT. Nel periodo precedente al lockdown eseguiva due trattamenti fisioterapici settimanali con obiettivi di facilitazione al controllo motorio, acquisizione di autonomia posturale, utilizzo degli arti superiori, implementazione della comunicazione e della relazione. Era anche seguito presso l'Ambulatorio del Bambino Complesso ( $\mathrm{ABC}$ ) per gli aspetti nutrizionali e funzionali legati all'alimentazione.

Dopo la chiusura dei servizi ambulatoriali, sono stati organizzati colloqui telefonici da parte della FT e del NPI per monitorare l'andamento e suggerire attività da eseguire a domicilio. Quando la famiglia è riuscita ad acquisire la tecnologia indispensabile, è stata avviata l'attività di videochiamata per il trattamento fisioterapico e insieme al pediatra dell'ABC si sono realizzati controlli della situazione clinica e verifiche del programma concordato durante l'ultimo controllo in presenza. Nei quattro mesi successivi, sono state effettuate videochiamate settimanali di circa 60 minuti l'una venendo incontro al desiderio della famiglia di proseguire il trattamento da remoto anche dopo la riapertura del mese di maggio.

Durante le videochiamate la FT propone alla madre di Antonio gli esercizi che vengono osservati e corretti contestualmente mentre la madre stessa li esegue. Contemporaneamente, sono verificate la correttezza della postura e l'adeguatezza degli ausili impiegati (sistema di postura seduta, passeggino posturale).

Gli ambiti su cui si è lavorato sono stati:

$>$ posturale/motorio con manipolazioni e attività di stretching, esercizi di controllo del capo e del tronco, controllo delle posture e degli ausili;

$>$ manipolazione con facilitazione al reaching e all'afferramento, manipolazione di oggetti di diverse dimensioni e consistenze e stimolazioni propriocettive;

> comunicazione, lavorando sulla causa-effetto, sulla risposta "sì e no", sull'inseguimento di stimoli target e sull'evocazione di risposte uguali a stimoli simili.

La FT ha mantenuto i contatti telefonici con l'équipe curante per ulteriori proposte di aggiornamento terapeutico e con videochiamate dirette per una valutazione multidisciplinare del bambino.

so il trattamento diretto grazie alla consolidata condivisione di compiti ed esercizi da riproporre al domicilio.

La fisioterapista ha dato maggiore spazio alle videochiamate in modalità sincrona, che hanno permesso la continuità del trat- tamento diretto grazie anche al coinvolgimento dei genitori che, guidati dal terapista, realizzavano gli esercizi sul e con il bambino.

L'attività degli educatori professionali ha richiesto la preparazione e la condivisione 
del materiale con la famiglia, in largo anticipo rispetto alla seduta, soprattutto là dove il contatto interpersonale, la mancanza di sguardo condiviso e le difficoltà di aggancio costituivano un oggettivo impedimento.

Nei primi mesi successivi al lockdown, le limitazioni persistenti hanno consentito di garantire un solo trattamento settimanale in presenza. Si è quindi proseguito anche con il trattamento da remoto nei casi in cui fosse previsto un secondo trattamento settimanale; la famiglia preferisse non accedere direttamente al servizio o nelle situazioni di prescritta quarantena. Durante questo periodo, 319 pazienti (per un totale di 786 prestazioni) hanno usufruito di questa modalità di trattamento (videochiamata o colloquio telefonico).

Intervistati con un questionario di gradimento, i genitori hanno espresso una valutazione complessivamente positiva dell'esperienza, con un punteggio medio di 8 su una scala da 0 a 10 . Le prestazioni in teleassistenza sono state valutate molto utili nella prosecuzione del percorso di cura, con un gradimento sovrapponibile alle visite tradizionali, ed è stata espressa la volontà di potere proseguire con una modalità integrata tra le due metodiche. La valutazione degli operatori ha restituito un punteggio medio pari a 6,7 su una scala da 0 e 10 . La maggioranza degli intervistati ha valutato positivamente l'interazione in teleassistenza, pur ritenendola meno efficace della modalità tradizionale. Anche gli operatori concordano nel prospettare la prosecuzione del servizio in teleassistenza integrato con le visite tradizionali, anche se individuano la prevalente utilità dei servizi telematici nella conduzione di riunioni, nella discussione di casi clinici e per la formazione. L'esperienza è stata giudicata più positivamente dagli operatori più giovani e con meno anni di servizio.

\section{Conclusioni}

Dall'esperienza vissuta nei mesi più critici della pandemia, molti spunti possono essere colti e utilizzati per arricchire la nostra pratica quotidiana in una prospettiva futura. Pur riconoscendo - e su questo operatori e utenti concordano - che il trattamento riabilitativo in presenza offra indiscutibili vantaggi, siamo tuttavia convinti che l'attività da remoto ci abbia regalato qualche nuova opportunità. Per il fisioterapista è stata la supervisione sui setting domiciliari, per l'educatore un diverso lavoro sulle autonomie e per il logopedista l'utilizzo delle risorse informatiche e delle piattaforme online. Anche i pazienti e le loro famiglie hanno apprezzato alcuni ambiti di interazione ancora inesplorati. L'assen-

TABELLA 2. Pazienti in trattamento riabilitativo da gennaio a luglio 2020

Numero pazienti in trattamento

\begin{tabular}{|l|c|c|c|}
\multirow{2}{*}{\multicolumn{1}{|c|}{ Diagnosi - ICD10 }} & \multicolumn{3}{c|}{ Numero pazienti in trattamento } \\
\cline { 2 - 4 } & $\mathbf{1 / 1 - 8 / 3}$ & $\begin{array}{c}\mathbf{9 / 3 - 3 1 / 5} \\
\text { (LOCKDOWN) }\end{array}$ & $\mathbf{1 / 6 - 3 1 / 7}$ \\
\hline Ritardo mentale lieve - F70 & 13 & 13 & 8 \\
\hline Ritardo mentale medio - F71 & 9 & 10 & 7 \\
\hline Disturbo del linguaggio - F80 & 165 & 143 & 165 \\
\hline Disturbi specifici dell'apprendimento - F81 & 39 & 15 & 37 \\
\hline Disprassia - F82 & 29 & 21 & 26 \\
\hline Disturbo evolutivo misto - F83 & 27 & 23 & 20 \\
\hline Autismo - F84 & 80 & 85 & 85 \\
\hline Epilessia - G40 & 11 & 14 & 10 \\
\hline Paralisi cerebrale infantile-G80 & 15 & 16 & 17 \\
\hline Sordità - H90 & 20 & 17 & 16 \\
\hline Sindrome di Down - 090 & 15 & 17 & 17 \\
\hline Totale & 423 & 374 & 408 \\
\hline
\end{tabular}

TABELLA 3. Prestazioni da remoto effettuate nel periodo 9/3/2020-31/5/2020

\begin{tabular}{|c|c|c|c|}
\hline Prestazione & Logopedista & Fisioterapista & $\begin{array}{c}\text { Educatore } \\
\text { professionale }\end{array}$ \\
\hline $\begin{array}{l}\text { Discussione del caso } \\
\text { e/o predisposizione del piano } \\
\text { di trattamento }{ }^{1}\end{array}$ & 735 & 275 & 264 \\
\hline Colloquio in videochiamata ${ }^{2}$ & 808 & 514 & 64 \\
\hline Colloquio telefonico significativo ${ }^{3}$ & 513 & 393 & 103 \\
\hline Totale & 2056 & 1182 & 431 \\
\hline
\end{tabular}

${ }^{1}$ Attività di preparazione del materiale e delle sedute di trattamento sia in modalità sincrona che asincrona.

${ }^{2}$ Trattamento effettuato con videochiamata in modalità sincrona.

${ }^{3}$ Colloqui telefonici di confronto con le famiglie in modalità asincrona.

za dell'operatore dal setting riabilitativo ha portato i genitori a mettersi maggiormente in gioco, a sviluppare nuove competenze tecniche, creative e relazionali e a diventare protagonisti, insieme al bambino, sperimentando una maggiore partecipazione al programma riabilitativo. Anche altre attività di supporto (riunioni di équipe, formazione, restituzioni alla famiglia, rapporto con enti esterni), svolte da remoto, sono state valutate positivamente perché hanno consentito un significativo risparmio di tempi e risorse che è stato possibile reimpiegare nell'assistenza ai pazienti. Il trattamento a distanza diventa un'ulteriore opportunità per i pazienti più fragili che abbiano difficoltà negli spostamenti o per specifiche esigenze familiari.

Le criticità sollevate vertono principalmente sulla maggiore complessità della relazione interpersonale e sull'impossibilità di svolgere alcune attività che non possono prescindere dall'intervento diretto del riabilitatore. La disponibilità per i servizi e per le famiglie di adeguati strumenti informatici è evidentemente un fattore limi- tante che deve essere previsto e superato. Se transitare dal trattamento in presenza a quello a distanza è stato generalmente agevole con le famiglie con le quali il rapporto di fiducia era ben consolidato, più difficile è stato l'approccio con i pazienti di più recente conoscenza e presa in carico. In questi casi, la teleriabilitazione ha mostrato evidenti limiti e il trattamento si è dovuto circoscrivere alle situazioni cliniche meno complesse.

Riabilitatori e famiglie concordano nel riconoscere che questa nuova modalità di trattamento ha potenzialità di integrazione con il classico approccio in presenza che meritano di essere valorizzate per il futuro. Il ruolo abituale del riabilitatore come facilitatore di buone prassi si arricchisce, quindi, di un ulteriore strumento in grado di garantire continuità al percorso assistenziale e riabilitativo del paziente e della sua famiglia.

\section{monica.giorgioni@auslromagna.it}

\section{La bibliografia è consultabile online.}




\section{Bibliografia}

1. Ameis SH, Lai M-C, Mulsant BH et al. Coping, fostering resilience, and driving care innovation for autistic people and their families during the COVID-19 pandemic and beyond. Mol Autism 2020;11:61.

2. Smile SC. Supporting children with autism spectrum disorder in the face of the COVID-19 pandemic. CMAJ 2020;192:E587.

3. Liu JJ, Bao Y, Huang X, et al. Mental health considerations for children quarantined because of COVID-19. Lancet Child Adolesc Health 2020;4:347-9.

4. Capello F. La telepediatria: prime indicazioni operative dagli USA. Quaderni acp 2018;25:30-2.

5. Magazzù G. Telemedicina: scelta obbligata o opportunità offerta dalla pandemia? Quaderni acp 2020;27;146.

6. Camden C, Pratte G, Fallon F, et al. Diversity of practices in telerehabilitation for children with disabilities and effective intervention characteristics: results from a systematic review. Disabil Rehabil 2019 ;Apr 12:1-13.

7. Corti C, Oldrati V, Oprandi MC, et al. Remote technology-based training programs for children with acquired brain injury: A systematic review and a meta-analytic exploration. Behav Neurol 2019;2019:1346987.

8. Glegg S. Virtual rehabilitation with children: challenges for clinical adoption [from the field]. IEEE Pulse. 2017;8:3-5.

9. Physiopedia. Evidence based practice (EBP) in physiotherapy. www.physio-pedia.com

10. Peretti A, Amenta F, Tayebati SK, et al. Telerehabilitation: review of the state-of-the-art and areas of application». JMIR Rehabil Assist Technol 2017;4:e7.

11. Park M, Giap TT, Lee M, et al. Patient- and family-centered care interventions for improving the quality of health care: A review of systematic reviews. Int J Nurs Stud 2018; 87:69-83.

12. Wang CJ, Ma J, Zuckerman B et al. The opportunities for telehealth in pediatric practice and public health. Pediatr Clin North Am 2020;67:603-11. 